\title{
EDITORIAL
}

\section{Virtual Is the New Reality}

\section{American Society for Investigative Pathology Scientific Meetings, Conferences, and Events in the Age of Coronavirus Disease 2019}

\author{
Chhavi Chauhan, ${ }^{*}$ William B. Coleman, ${ }^{*}$ and Richard N. Mitchell ${ }^{\dagger}$
}

\begin{abstract}
From the American Society for Investigative Pathology,* (Director of Scientific Outreach and Executive Officer, respectively) Rockville, Maryland; and the Lawrence J. Henderson Professor of Pathology, ${ }^{\dagger}$ Department of Pathology, Brigham and Women's Hospital and Harvard Medical School, Boston, Massachusetts (Senior Associate Editor)
\end{abstract}

\section{The Year of the Pandemic}

The year 2020 will forever be remembered as the year the severe acute respiratory syndrome coronavirus 2 [SARSCoV-2; coronavirus disease 2019 (COVID-19)] pandemic came to the United States and the world, with devastating consequences. As of this writing (late December 2020), the United States has recorded nearly 18 million cases of COVID-19 and $>315,000$ deaths, and there have been nearly 77 million cases and 2 million deaths worldwide (Johns Hopkins University of Medicine, Coronavirus Resource Center, https://coronavirus.jhu.edu/map.html, last accessed December 21, 2020). The pandemic at first caused ripples and then giant waves with respect to altering the normality of life and work as states and cities attempted to combat viral spread by closing offices and businesses, and implementing public health mitigation efforts. Academic institutions and research laboratories were also not spared, and many if not most research laboratories closed for a period of time (months) and then slowly reopened with new restrictions. The short-term impacts were felt by graduate students and postdoctoral fellows, many of whom switched to remote learning for their classes and worked from home on their research (to the extent possible). For those who were fortunate to continue working in their laboratories, heightened use of personal protective equipment was mandated, making work more difficult and uncomfortable. Longer-term effects are anticipated for principal investigators who consumed grant funds to support their personnel even as productivity necessarily declined with the closing of laboratories. Of course, the overall impact on research progress is not yet known, and will not be known for some time. On a positive note, the closing of research laboratories, mandated use of personal protective equipment, and implementation of public health mitigation efforts provided some measure of protection for the research workforce.

Scientific societies have not been spared the effects of the COVID-19 pandemic. Organizations like the American Society for Investigative Pathology (ASIP) have been fortunate to continue operations, but with dramatic changes in how we accomplish what we do. In the case of the ASIP, necessity has been the driver of implementation. Before the beginning of the year, the ASIP had many plans on the drawing board for future execution. With the advent of the pandemic, these plans were prioritized and advanced into final form. As one might expect, all of the new activities and initiatives share the common element of existing in the virtual realm. Although the pandemic has prevented us from meeting each other in the typical face-to-face manner to which we are accustomed, the society has found ways to connect with members virtually. Although we might not be pioneers of the virtual lecture or the virtual meeting, we have introduced numerous innovations into our virtual events, making them more effective and useful to our

Accepted for publication December 10, 2020.

Disclosures: None declared.

Address correspondence to Chhavi Chauhan, Ph.D., American Society for Investigative Pathology, 1801 Rockville Pike, Ste. 350, Rockville, MD 20852. E-mail: cchauhan@asip.org. 
members and colleagues. This editorial describes in some detail several new initiatives the ASIP has undertaken since the beginning of the pandemic.

\section{Virtual Lectures, Webinars, and Other Events}

In the spring of 2020, multiple forces combined to drive the development of an ASIP webinar series. The 2020 ASIP Annual Meeting was canceled because of the COVID-19 pandemic, generating a scientific void, and there was a general desire on the part of our members to learn about the SARS-CoV-2 virus and associated diseases. Furthermore, numerous ASIP members became engaged in COVID-19 research, investigating the pathology, pathogenesis, and pathophysiology of this viral disease. Hence, with a ready audience and a cadre of experts to draw upon, the ASIP embarked on the path of virtual learning with the launch of the COVID-19 webinar series (https://asippathways.com/ webinars) in June 2020. The overarching goal of the webinar series was to keep the membership, as well as other biomedical researchers, physicians, and frontline health care workers, abreast of the fast-evolving developments in the field of COVID-19. To date, the webinar series has covered a range of timely topics, including but not limited to COVID-19 pathology (based on autopsy findings) and underlying disease mechanisms, pathophysiology of patients with COVID-19 (such as the cytokine storm), and potential therapeutic interventions and treatments (such as convalescent plasma therapy), as well as challenges associated with molecular and serologic testing for detection of SARS-CoV-2. By way of innovation, these webinars typically feature multiple expert panelists who share their observations, experiences, and supporting data, followed by discussion moderated by the ASIP Director of Scientific Outreach. The inclusion of complimentary experts who bring various viewpoints, provides the audience with an enhanced perspective of the topic, and the inclusion of an ample moderated discussion period enables questions to be answered to further enhance the event. The response to these timely and educational webinars has been gratifying and overwhelming at the same time. We have received rave reviews, stunning testimonials, as well as exciting feedback, which continue to guide our efforts to develop additional webinars on COVID-19-related topics. We also anticipate development of webinars on other scientific topics, some in collaboration with other scientific societies.

With the early success of the COVID-19 webinar series, plans were developed for additional virtual events during the summer and fall of 2020. Given the cancellation of the Annual Meeting, the meritorious award lectures were held as stand-alone virtual events (https://asippathways.com/ 2019/09/25/asip-2020-meritorious-award-lectures). These award lectures featured the investigators who received the 2020 meritorious awards: the ASIP Rous-Whipple Award, the ASIP Outstanding Investigator Award, the ASIP Cotran
Early Career Investigator Award, and the ASIP Young Scientist Leadership Award. By holding these meritorious award lectures virtually, the audience base could be expanded to both members and nonmembers, and individuals who typically do not attend the Annual Meeting. Hence, the meritorious research of several ASIP members was showcased to a diverse audience, potentially attracting new members into the society.

The ASIP also took advantage of the virtual setting to promote connections among the various communities within our membership. The Women in Pathology (https://www.asip. org/membership-community/women-in-pathology) held an interactive networking session that enabled their leadership to engage with its members to discuss their goals and objectives. The Liver Pathobiology Scientific Interest Group (https://www.asip.org/membership-community/scientificinterest-groups/liver-pathobiology) held a virtual Meetthe-Expert session, featuring short talks by two experts and an interactive discussion. Finally, the trainee members of the society held several Virtual Trainee Hangouts, in which the moderators led discussion of various issues, including the impact of the COVID-19 pandemic on the training progress of graduate students and postdoctoral fellows, and the associated communication challenges.

\section{Virtual Scientific Meetings: PISA 2020 Virtual and Interactive}

Well before COVID-19 became a global concern, as early as April 2019, a cabal of Boston, MA, ASIP members began sketching out the outlines of a Pathobiology for Investigators, Students, and Academicians (PISA) scientific get-together that would live up to the high standards for scientific meetings previously established in the 2015 to 2018 PISA meetings held in Bethesda, MD, Houston, TX, Pittsburgh, PA, and Ann Arbor, MI. As the signature stand-alone meeting for ASIP, it was important that a Boston PISA-especially one hosted in such a researchintensive environment-be a gold-star 3-day event, jampacked with academic and scientific luminaries, as well as a chance to showcase the city. Studiously avoiding overlaps with scientific meetings promoted by other societies, a date was set, sessions were crafted, venues were scouted, and speakers were invited by members of the Organizing Committee (https://pisa20.asip.org/about-pisa-2020/pisa2020-planning-committee). The only coronavirus that anyone even vaguely knew about was SARS from almost two decades prior. By late winter (in early 2020), an outstanding scientific program was in place, and plans were being finalized $\ldots$ and then COVID-19 came to town-indeed, around the world. For a period of months, the planning committee and ASIP leadership remained optimistic that we would still be able to convene in person. However, as spring became summer, it became increasingly clear that federal, Massachusetts state, and Boston city 
guidelines and restrictions would necessitate implementing "Plan B"- an all-virtual meeting.

At that point, Lisa McFadden (ASIP Director of Scientific Meetings, Membership, and Educational Services) and other ASIP staff swung into action. The meeting had to be completely reconfigured from all-day live plenaries, concurrent symposia, and abstract-driven talks, largely taking place over a single weekend, to five afternoon sessions occurring virtually across the work week. An online meeting company was engaged, and a virtual platform was developed to accommodate all the moving parts of a PISA meeting: speakers, posters, Scientific Interest Groups, special sessions, mentoring, and networking events. All the speakers needed to be rescheduled, and all the advance publicity needed to be reworked. In addition, the exceptional scientific program was significantly enhanced to provide special sessions for trainees, Women in Pathology, and Scientific Interest Groups (https://pisa20.asip.org/ virtual-program/virtual-program). Although initially disappointing, switching from an in-person to an allvirtual event actually had several significant silver linings. For starters, there was no time away from laboratories, academic institutions, and hospital responsibilities, and no travel and housing costs; speakers and attendees were saved the wear and tear of physically getting to New England, and everyone could safely take part from the comfort of their own homes or offices. Another benefit of a virtual meeting was that with every session being recorded, attendees could view select sessions and speakers on demand, on a schedule that worked for them. Instead of blocking out time for poster presenters to stand by their work, poster sessions could be viewed asynchronously, with interested parties able to engage when it was convenient. Although the camaraderie and live connections were certainly missed, virtual events were scheduled for attendees to socialize and network with colleagues. There was also a financial benefit for the society in that a virtual meeting is less expensive to implement than one held in person. Indeed, the society allowed ASIP member trainees to attend for free, and everyone else participated at a significantly reduced registration rate. The virtual nature of the meeting with the associated cost savings was also a significant opportunity for attracting new eyes to the ASIP. The one significant downside was that with laboratories shutting down earlier in the year, many groups felt that they did not have the research advances necessary to merit submitting an abstract. The meeting organizers recognized this and worked with investigators to develop a uniform approach to sharing a blend of recent research and incremental developments.

Although it is likely that the society will return to inperson meetings once the dust has cleared and SARS-CoV-2 is in our rearview mirror, we may actually retain some of the features of the online virtual experience during future traditional events. Indeed, hybrid meetings-with some attendees in person and others Zoomed in-may become the new norm. Hybrid meetings will certainly expand opportunities for those who might have timing or financial issues; trainees could have the benefit of participating in a first-class meeting without breaking the principal investigator's bank. It would also markedly expand the opportunities to bring in speakers from anywhere-without incurring the cost of travel and housing. In unexpected ways, a global pandemic may have given rise to truly global meetings.

\section{Virtual Annual Meeting at Experimental Biology 2021}

The ASIP Annual Meeting takes place during the Experimental Biology meeting each spring in conjunction with four other Experimental Biology scientific societies and several guest societies. As mentioned, the COVID-19 pandemic caused the cancellation of the 2020 Annual Meeting. By summer of 2020, the society meeting planners decided that the Experimental Biology 2021 meeting (https://experimentalbiology.org/Home), and thereby the 2021 ASIP Annual Meeting (http://asip2021.asip.org), should be completely virtual. This decision reflects the likelihood that by May 2021, local and state restrictions in the Indianapolis, IN, area would not permit a gathering as large as the Experimental Biology meeting, and the fact that many of our members may be hesitant to travel and attend such a large meeting. Although Experimental Biology 2021 will not be the first large meeting to be held virtually, it will be the first large virtual meeting for the ASIP and sister societies. The ASIP will work hard to ensure that the 2021 Annual Meeting contains all of the elements of an outstanding scientific meeting with abundant opportunities for our members and colleagues to present their research, attend career development and educational sessions, and engage in networking events. The Program Committee (https://www.asip.org/about-asip/ committees/program-committee), the Education Committee (https://www.asip.org/about-asip/committees/educationcommittee), the Committee for Career Development and Diversity (https://www.asip.org/about-asip/committees/ committee-for-career-development-and-diversity), and several Scientific Interest Group (https://www.asip.org/ membership-community/scientific-interest-groups) leaders are already heavily engaged in planning the various sessions and events for the meeting. The success of the virtual Experimental Biology 2021 meeting will require all of the effort associated with a face-to-face meeting, as well as an innovative approach and the introduction of new elements to enhance the excellent scientific program that has been developed. We look forward to the great opportunity that a virtual Experimental Biology 2021 presents, and will experiment with virtual sessions and events that might be incorporated into future hybrid meetings. A primary objective is to increase the level of 
interaction despite the virtual separation of our members during the meeting.

\section{Silver Linings and Golden Opportunities}

As described herein, the COVID-19 pandemic served as a major driver for the implementation of numerous new virtual sessions and events for ASIP members and our colleagues in the field of pathobiology research. The ASIP is a community of investigators who study disease. Everything we do is designed to enhance the community of members overall, or smaller communities represented by subsets of our membership. The newly developed and implemented virtual sessions and events facilitate the interaction and engagement of members of all of these communities at times and places where these interactions and engagements did not previously exist. We are accustomed to seeing each other face-to-face at the Annual Meeting and/or at PISA, with other interactions during the year being confined largely to committee work. Now, out of pandemic-related necessity and through innovation, we have generated new opportunities in the virtual space for the members of the ASIP to meet and share their research, network, and expand their knowledge, resulting in stronger and more engaged communities within the ASIP membership. In the virtual space, the possibilities are endless. The society looks forward to continuing with virtual events far into the future and implementation of additional virtual opportunities for our members and the scientific community at large. By enhancing the communities within the ASIP, we will promote our individual members and their work, and will benefit biomedical science at the same time. 